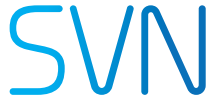

Stroke \& Vascular Neurology

\section{Would integrated Western and traditional Chinese medicine have more benefits for stroke rehabilitation? A systematic review and meta-analysis}

To cite: Zhong LLD, Zheng Y, Lau AY, et al. Would integrated Western and traditional Chinese medicine have more benefits for stroke rehabilitation? A systematic review and metaanalysis. Stroke \& Vascular Neurology 2022;7: e000781. doi:10.1136/svn-2020-000781

- Additional supplemental material is published online only. To view, please visit the journal online (http://dx.doi.org/10. 1136/svn-2020-000781).

LLZ and YZ are joint first authors.

Received 8 December 2020 Accepted 2 August 2021 Published Online First 26 August 2021
Check for updates

(C) Author(s) (or their employer(s)) 2022. Re-use permitted under CC BY-NC. No commercial re-use. See rights and permissions. Published by BMJ.

For numbered affiliations see end of article.

Correspondence to Dr Linda LD Zhong; Idzhong@hkbu.edu.hk

Dr. Alexander Y Lau; alexlau@cuhk.edu.hk

\section{ABSTRACT}

Background Stroke is a major cause of death or longterm disability worldwide. Many patients with stroke receive integrative therapy consisting of Western medicine (WM) and routine rehabilitation in conjunction with Chinese medicine (CM), such as acupuncture and Chinese herbal medicine. However, there is no available evidence on the effectiveness of the combined use of WM and CM interventions in stroke rehabilitation.

Aims The purpose of this meta-analysis is to evaluate the results of all individual studies to assess the combined use of CM and WM in stroke rehabilitation compared with WM only.

Methods The Preferred Reporting Items for Systematic Reviews and Meta-Analysis guidelines were followed. MEDLINE, EMBASE, Cochrane and China National Knowledge Infrastructure (CNKI) were searched. The included outcomes were dependency, motor function, depression and swallowing function. Subgroup analysis was performed, and publication bias was assessed using funnel plots.

Summary of review 58 studies and 6339 patients were included in the meta-analysis. Subgroup analysis revealed that combined therapy comprising both acupuncture and WM had a superior effect on improving dependency and swallowing function compared with standard WM therapy alone. Potential superiority of combined therapy comprising $\mathrm{CM}$ and WM in improving depression compared with standard WM therapy was also found.

Conclusions Our results indicate that the combined use of CM and WM could be more efficacious in stroke rehabilitation compared with the use of WM therapy alone. However, most studies were short in duration (2 to 4 weeks) and prone to different types of biases, which prevents making any conclusion regarding the long-term effects and raises concerns regarding true efficacy in context of high likelihood of Hawthorn bias. So, more randomised controlled trials with more rigorous design and longer duration of treatment and follow-up need to be conducted to compare WM alone versus WM and CM combined.

PROSPERO registration number CRD42020152050.

\section{INTRODUCTION}

In China, many patients with stroke receive integrative medicine, which include treatment by Western medicine (WM) and routine rehabilitation in conjunction with acupuncture and/or Chinese medicine (CM).$^{12}$ The CM-based rehabilitation is characterised by holistic concepts with multiple therapeutic approaches, mostly comprising acupuncture and herbal medicine. ${ }^{34}$ Chinese herbal medicine (CHM) has multifactorial effects, which include antioxidant, anti-inflammatory antiapoptotic, neuroprotective and vascular protective properties, and it is believed to be efficacious in stroke treatment. ${ }^{5}$ Regarding acupuncture, available evidence suggests that it may have beneficial effects on improving dependency, global neurological deficiency and some specific neurological impairments in people with stroke in the convalescent stage as well as no obvious adverse events. ${ }^{67}$

Despite clinical studies suggesting that integrative medicine is effective in stroke rehabilitation with regards to improving dependency, motor function, depression and swallowing function, the evidence generally is low in quality. ${ }^{8-11}$ A systematic review to estimate the efficacy of integrative medicine and summarise the overall quality of existing clinical studies is, therefore, essential to gain insight and posit a framework for the development of integrated clinical services. As such, the purpose of this systematic review is to summarise the results of all clinical trials using combined $\mathrm{WM}$ and $\mathrm{CM}$ interventions in terms of improvements in the outcomes of dependency, motor function, depression and swallowing function during stroke rehabilitation.

\section{METHODS}

This systematic review with meta-analysis was performed according to the Preferred Reporting Items for Systematic Reviews and Meta-Analysis guidelines. ${ }^{12}$ The study 
protocol was registered at PROSPERO prior to the start of the review (https://www.crd.york.ac.uk/prospero/display record.php?RecordID=152050).

\section{Search strategy}

We searched MEDLINE, EMBASE, the Cochrane Central Register of Controlled Trials (CENTRAL) and the China National Knowledge Infrastructure (CNKI) from inception to January 2021 using medical subject headings and text words related to stroke rehabilitation. We referred to the published Cochrane protocol about $\mathrm{CM}$ and stroke rehabilitation to initiate our search strategies. ${ }^{13-15}$ The detailed search strategy used is described in online supplemental table 1.

\section{Selection criteria}

We included randomised controlled trials (RCTs) and quasi-RCTs on stroke rehabilitation that compared WM and CM interventions (mainly CHM, acupuncture, Tuina and Moxibustion) with at least one control group only using WM practice. In the quasi-RCTs, participants were allocated to different arms of the trial using a method of allocation that was not completely random, such as admission sequence. Both parallel groups and crossover designs were included. The main stroke types included were ischaemic stroke and haemorrhagic stroke. Trials involving participants of any age or sex during poststroke rehabilitation (14 days since onset) were included.

The included outcomes were dependency, motor function, depression and swallowing function. For the assessment of dependency, the Barthel Index, Modified Barthel Index, activities of daily living and Comprehensive Functional Assessment were included. Regarding motor function, the Fugl-Meyer Assessment, Brunnstrom recovery stages score and motor evoked potential were included. The included assessments to evaluate improvement in depression were the Hamilton depression rating scale, Hamilton anxiety rating scale, Symptoms of Traditional Chinese Medicine depression scale and 10-item Center for Epidemiological Studies Depression Scale. Swallowing function was measured by the water drinking test, standardised swallowing assessment and repetitive salivaswallowing test.

We excluded case-control, cohort and cross-sectional studies and studies with sample size of less than 50 participants.

\section{Data extraction and outcomes}

A first screening was performed by seven independent screening reviewers based on the titles and abstracts of all articles found by the database searches (after removal of duplicates). Some obviously irrelevant studies, such as non-clinical trials, animal studies and those without poststroke rehabilitation outcomes, were excluded. Any discordance was resolved by discussion among all seven reviewers. After the first screening, preliminary included articles were screened based on their full texts by two reviewers (YZ and LLDZ). They were further assessed according to our inclusion and exclusion criteria, and the eligible studies were included in the meta-analysis. The other authors, NW, XW, and TS, conducted literature search and synthesised the data. ZL and HL extracted the data and produced the figures. Rayyan was used for gathering all papers, removing duplicates and performing article screening. ${ }^{16}$ Information was extracted from each included study regarding the study design, sample size, condition, characteristics of included participants, type of intervention (including type, dose, duration and frequency of treatment in various intervention groups) and outcomes.

\section{Risk of bias assessment}

To ascertain the validity of eligible randomised trials, two review authors (NW and LY) assessed potential risks of bias independently for all included studies using the Cochrane's tool for assessing risk of bias. ${ }^{17}$ They assessed all six domains (sequence generation; allocation concealment; blinding of participants, personnel and outcome assessors; incomplete outcome data; selective outcome reporting and other sources of bias) for each study and assigned a score (high, low or unclear) depending on their own respective judgement. The criteria used for assessing the risk of bias are shown in the online supplemental. We contacted the study authors for clarification if necessary in cases of potential risks of bias.

\section{Statistical synthesis and analysis}

For continuous outcomes, considering the various scales used, we used both standard mean difference (SMD) and weighted mean differences with 95\% CI. RevMan V.5.3 software offered by Cochrane collaboration was used for all data analyses. Forest plots were generated to visually assess the effect sizes and corresponding 95\% CIs using random effects models. We conducted a subgroup analysis of short-term duration ( $\leq 1$ month) versus longer term duration ( $>1$ month), hypothesising a larger effect in the short-term group. $\mathrm{P}<0.05$ was considered as significant for the subgroup effect.

Funnel plots were adopted to assess the possibility of publication bias. The tools were applied only when at least 10 studies reported the patient important outcomes, since the power of the tests would be too low to distinguish chance from real asymmetry for fewer studies. ${ }^{17}$ The Grading of Recommendations Assessment, Development and Evaluation (GRADE) approach was used to assess the quality of the findings of outcomes. ${ }^{18}$ Certainty of evidence was divided into four levels: high quality: further research is very unlikely to change our confidence in the estimate of effect; moderate quality: further research is unlikely to have an important impact on our confidence in the estimate of effect and may change the estimate; low quality: further research is very likely to have an important impact on our confidence in the estimate of effect and is likely to change the estimate and very low quality: we are very uncertain about the estimate. ${ }^{19}$ 




Figure 1 Flow diagram of study selection. RCT, randomised controlled trial.

\section{RESULTS}

\section{Study characteristics}

We screened the titles and/or abstracts of 8126 studies and excluded 7106 irrelevant studies. We then screened full texts of the remaining 1020 studies and excluded 726 studies that did not have full texts, covered animal research or did not cover RCTs, poststroke rehabilitation or CM. Finally, 294 studies were included in the qualitative synthesis. Among them, 236 studies were excluded as their sample size was less than 50 , and they did not contain a suitable comparison of CM combined with WM versus only WM or relevant data. As such, 58 studies with 6339 patients in total were included in the meta-analysis. The detailed flow diagram is included in figure 1 .

The 58 included studies consisted of 7 quasi-RCTs and 51 RCTs. Among these studies, 14 reported the usage of a blinding method and 9 reported having a follow-up period after treatment. Moreover, 39 studies compared WM and acupuncture with WM, 14 studies compared WM and CHM with WM and 5 studies compared WM and other CM interventions (eg, Tuina, Moxibustion etc) with WM. Among the 58 studies, the 10 most commonly used CM herbs were Astragali Radix (Huangqi), Chuanxiong Rhizoma (Chuanxiong), Bupleuri Radix (Chaihu), Curcumae Radix (Yujin), Angelicae Sinensis Radix (Danggui), Poria (Fuling), Cinnamomi Ramulus (Guizhi), Pheretima (Dilong), Paeoniae Radix Alba (Baishao), and Pinelliae Rhizoma (Banxia). In addition, the 10 most commonly used acupoints were Hegu ( $L I 4)$, Baihui $(D U$ 20), Neiguan (PC O), Zusanli (ST 36), Fengchi (GB 20), Lianquan ( $R N$ 23), Sanyinjiao ( $S P$ O), Waiguan (SJ 5), Yanglingquan (GB 34) and Quchi (LI 11). Details of these 


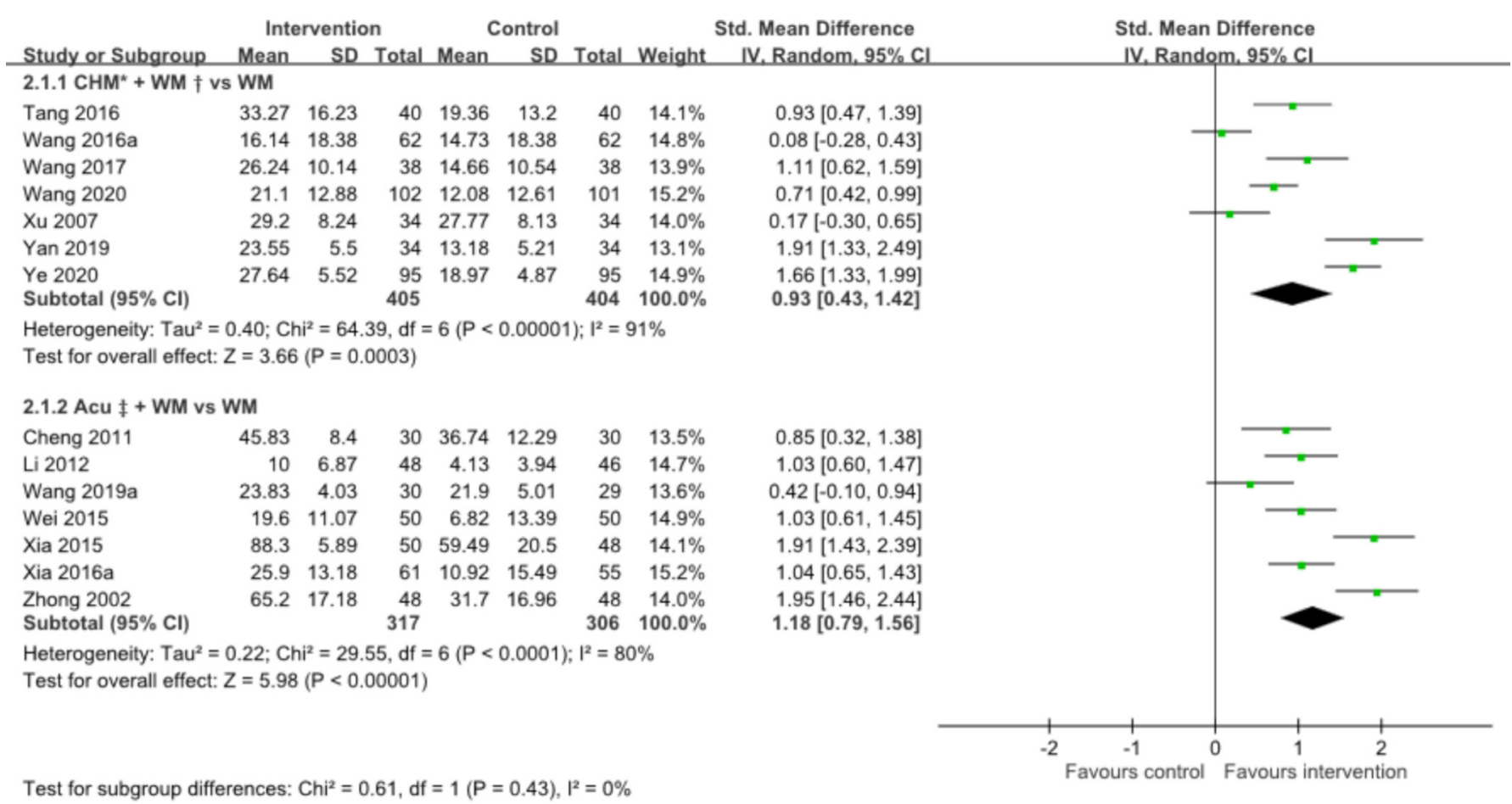

*CHM: Chinese Herbal Medicine; †WM: Western Medicine; $\$$ Acu: Acupuncture.

** The reference for each study have been listed in Supplement Table 2 and 'List of included studies' references'.

Figure 2 The improvement of integrated medicine compared with western medicine only in dependency.

are shown in the characteristics table in online supplemental table 2 .

The meta-analysis results of each assessment are shown in online supplemental figures $1-4$. The funnel plots of the outcomes and details about the risks of bias are shown in online supplemental figures $5-8$ and online supplemental figures 13 and 14 . The subgroup analysis includes studies comparing the efficiency of the combined use of WM with either CHM or Acupuncture versus the use of WM only. The results of each assessment were pooled based on their categories of outcomes and reported as SMDs and 95\% CIs in figures 2-5. And the GRADE summary of outcomes in the subgroup analysis is displayed in table 1. Furthermore, another subgroup analysis based on the duration of treatment is shown in table 2 .

\section{Improvement in dependency}

We stratified the included studies according to subgroups as shown in figure 2. Both subgroups showed the superiority of combined treatment compared with WM therapy only in the improvement of dependency. Seven RCTs displayed the superiority of the effect of CHM and WM combined therapy compared with WM therapy only (809 patients; SMD 0.93 , 95\% CI 0.43 to $1.42 ; \mathrm{I}^{2}=91 \%$ ). Six RCTs and one quasi-RCT displayed the superiority of the effect of acupuncture and WM combined therapy compared with standard WM therapy only (623 patients;
SMD $1.18,95 \%$ CI 0.79 to 1.56 ; $I^{2}=80 \%$, low quality, figure 2 and table 1 ).

\section{Improvement in motor function}

Subgroup analysis on the improvement of motor function was performed. We stratified the included studies according to subgroups as shown in figure 3. Both subgroups showed the superiority of combined treatment compared with baseline WM therapy in the improvement of motor function. Three RCTs showed the superiority of the effect of CHM and WM combined therapy compared with baseline WM therapy (473 patients; SMD 1.27, 95\% CI 0.61 to $1.93 ; \mathrm{I}^{2}=90 \%$, low certainty, figure 3 and table 1 ). Seven RCTs and one quasi-RCT showed the superiority of the effect of acupuncture and WM combined therapy compared with baseline WM therapy (976 patients; SMD $0.76,95 \%$ CI 0.32 to $1.20 ; I^{2}=90 \%$, low certainty, figure 3 and table 1).

\section{Improvement in depression}

Subgroup analysis on the improvement of depression was performed. We stratified the included studies according to subgroups as shown in figure 4 . Both subgroups showed the superiority of combined treatment compared with baseline WM therapy in the improvement of depression. Six RCTs and one quasi-RCT demonstrated the superiority of the effect of CHM and WM combined therapy compared with baseline WM therapy (641 patients; SMD 


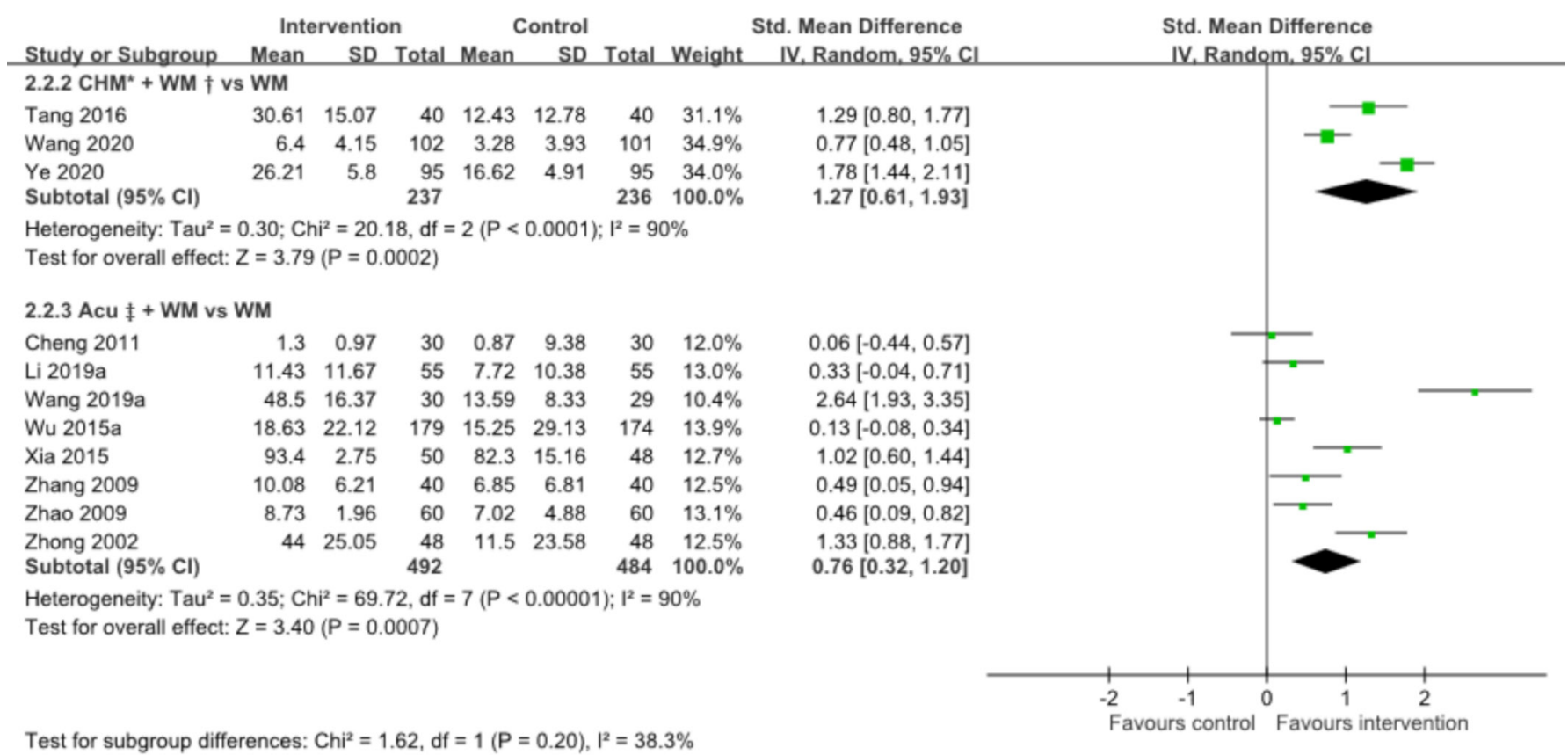

*CHM: Chinese Herbal Medicine; †WM: Western Medicine; $\$$ Acu: Acupuncture.

** The reference for each study have been listed in Supplement Table 2 and 'List of included studies' references'.

Figure 3 The improvement of integrated medicine compared with western medicine only in motor function.

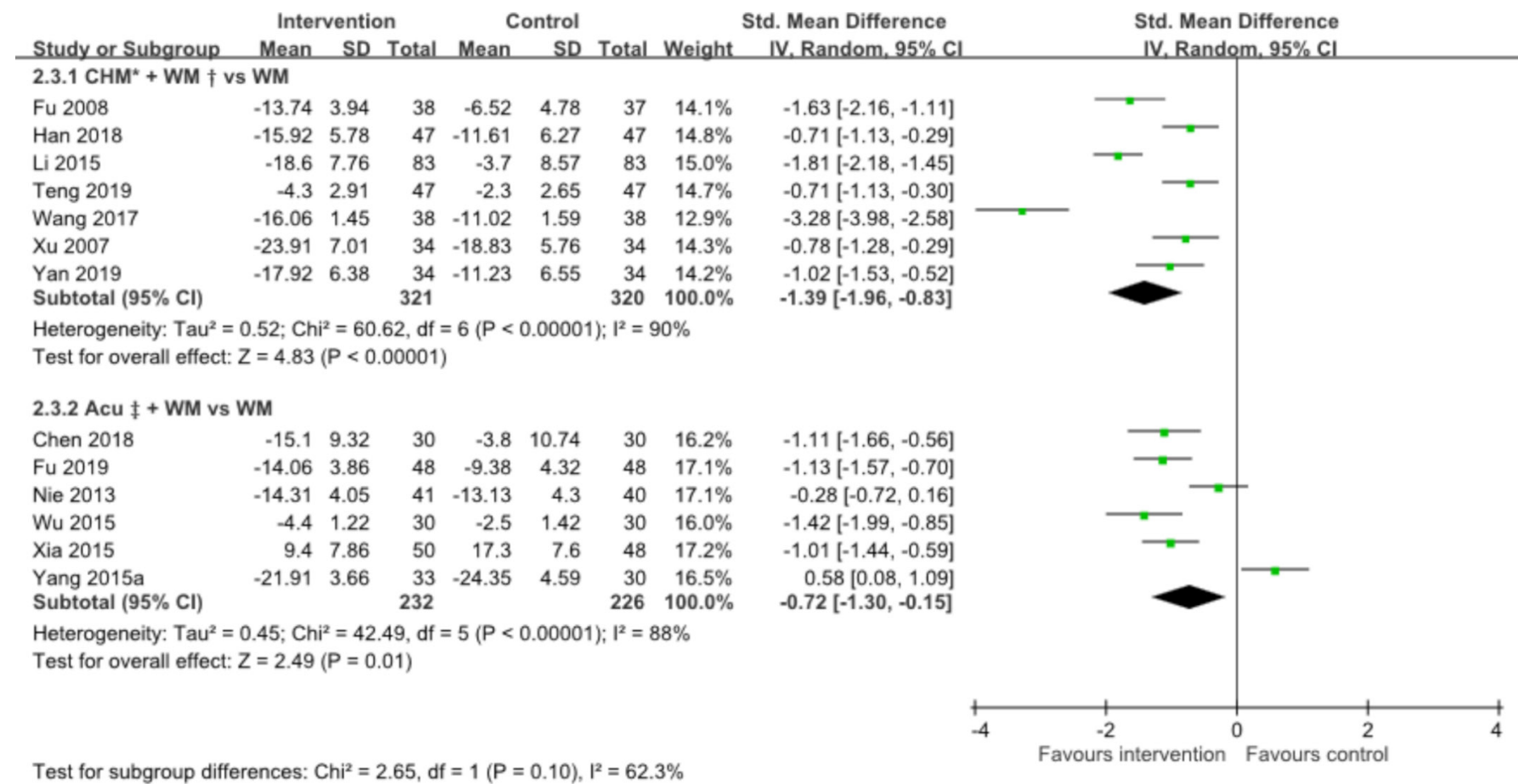

*CHM: Chinese Herbal Medicine; †WM: Western Medicine; $\$$ Acu: Acupuncture.

** The reference for each study have been listed in Supplement Table 2 and 'List of included studies' references'.

Figure 4 The improvement of integrated medicine compared with western medicine only in depression. 


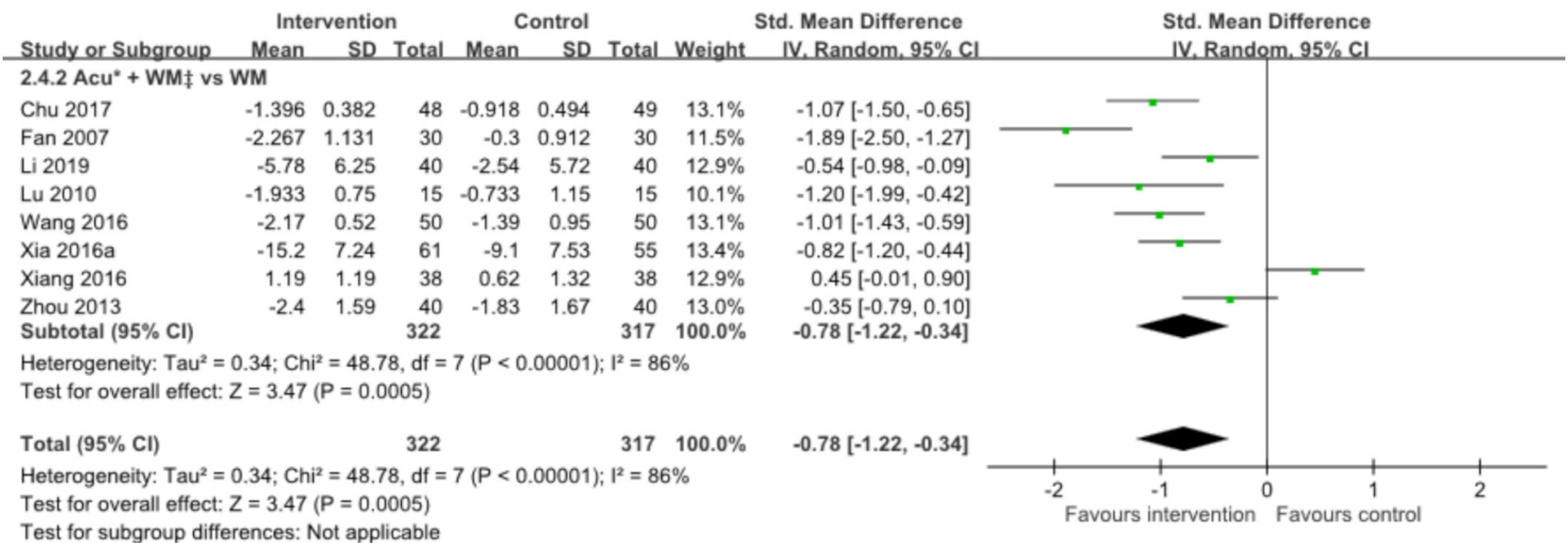

*Acu: Acupuncture; \$WM: Western Medicine.

** The reference for each study have been listed in Supplement Table 2 and 'List of included studies' references'.

Figure 5 The improvement of integrated medicine compared with western medicine only in swallowing function.

$-1.39,95 \%$ CI -1.96 to $-0.83 ; \mathrm{I}^{2}=90 \%$, low certainty, figure 4 and table 1). Six trials demonstrated the superiority of the effect of acupuncture and WM combined therapy compared with baseline WM therapy (458 patients; SMD $-0.72,95 \%$ CI -1.30 to -0.15 ; $\mathrm{I}^{2}=88 \%$, low certainty, figure 4 and table 1 ).

Table 1 GRADE‡ summary of findings of outcomes

\begin{tabular}{|c|c|c|c|}
\hline Outcomes & Effects $95 \% \mathrm{Cl}^{*}$ & Certainty of the evidencet & Interpretations \\
\hline $\begin{array}{l}\text { Improvement in } \\
\text { dependency } \\
\text { (CHM+WM vs WM) }\end{array}$ & $\begin{array}{l}\text { SMD } 0.9395 \% \mathrm{Cl}(0.43 \text { to } 1.42) \text {. } \\
\text { Based on data from } 809 \text { patients in } \\
\text { seven studies }\end{array}$ & $\begin{array}{l}\text { Low (serious risk of bias, } \\
\text { inconsistence) }\end{array}$ & $\begin{array}{l}\mathrm{CHM}+\mathrm{WM} \text { may improve } \\
\text { dependency }\end{array}$ \\
\hline $\begin{array}{l}\text { Improvement in } \\
\text { dependency } \\
\text { (Acuf+WM vs. WM) }\end{array}$ & $\begin{array}{l}\text { SMD } 1.1895 \% \mathrm{Cl}(0.79 \text { to } 1.56) \text {. } \\
\text { Based on data from } 623 \text { patients in } \\
\text { seven studies }\end{array}$ & $\begin{array}{l}\text { Low (serious risk of bias, } \\
\text { inconsistence) }\end{array}$ & $\begin{array}{l}\text { Acu +WM may improve } \\
\text { dependency }\end{array}$ \\
\hline $\begin{array}{l}\text { Improvement in motor } \\
\text { function } \\
\text { (CHM+WM vs. WM) }\end{array}$ & $\begin{array}{l}\text { SMD } 1.2795 \% \mathrm{Cl}(0.61 \text { to } 1.93) \text {. } \\
\text { Based on data from } 473 \text { patients in } \\
\text { three studies }\end{array}$ & $\begin{array}{l}\text { Low (serious risk of bias, } \\
\text { inconsistence) }\end{array}$ & $\begin{array}{l}\mathrm{CHM}+\mathrm{WM} \text { may improve } \\
\text { motor function }\end{array}$ \\
\hline $\begin{array}{l}\text { Improvement in motor } \\
\text { function } \\
\text { (Acu +WM vs. WM) }\end{array}$ & $\begin{array}{l}\text { SMD } 0.7695 \% \mathrm{Cl}(0.32 \text { to } 1.2) \text {. Based } \\
\text { on data from } 976 \text { patients in eight } \\
\text { studies }\end{array}$ & $\begin{array}{l}\text { Low (serious risk of bias, } \\
\text { inconsistence) }\end{array}$ & $\begin{array}{l}\text { Acu +WM may improve motor } \\
\text { function. }\end{array}$ \\
\hline $\begin{array}{l}\text { Improvement in depression } \\
\text { (CHM+WM vs. WM) }\end{array}$ & $\begin{array}{l}\mathrm{SMD}-1.3995 \% \mathrm{Cl}(-1.96 \text { to } 0.83) \text {. } \\
\text { Based on data from } 641 \text { patients in } \\
\text { seven studies }\end{array}$ & $\begin{array}{l}\text { Low (serious risk of bias, } \\
\text { inconsistence) }\end{array}$ & $\begin{array}{l}\mathrm{CM}+\mathrm{WM} \text { may improve } \\
\text { depression. }\end{array}$ \\
\hline $\begin{array}{l}\text { Improvement in depression } \\
\text { (Acu +WM vs. WM) }\end{array}$ & $\begin{array}{l}\text { SMD }-0.7295 \% \mathrm{Cl}(-1.30 \text { to } 0.15) \text {. } \\
\text { Based on data from } 458 \text { patients in six } \\
\text { studies }\end{array}$ & $\begin{array}{l}\text { Low (serious risk of bias, } \\
\text { inconsistence) }\end{array}$ & $\begin{array}{l}\text { Acu +WM may improve } \\
\text { depression. }\end{array}$ \\
\hline $\begin{array}{l}\text { Improvement in swallowing } \\
\text { function (Acu +WM vs. } \\
\text { WM) }\end{array}$ & $\begin{array}{l}\mathrm{SMD}-0.7895 \% \mathrm{Cl}(-1.22 \text { to } 0.34) \text {. } \\
\text { Based on data from } 639 \text { patients in } \\
\text { eight studies }\end{array}$ & $\begin{array}{l}\text { Low (serious risk of bias, } \\
\text { inconsistence) }\end{array}$ & $\begin{array}{l}\text { Acu +WM may improve } \\
\text { swallowing function. }\end{array}$ \\
\hline
\end{tabular}

${ }^{*} \mathrm{SMD}=0.2,0.5,0.8$ were considered as small effect, moderate effect and large effect, respectively.

†The certainty of evidence is divided into three levels: high quality: further research is very unlikely to change our confidence in the estimate of effect; moderate quality: further research is unlikely to have an important impact on our confidence in the estimate of effect and may change the estimate; low quality: further research is very likely to have an important impact on our confidence in the estimate of effect and is likely to change the estimate; very low quality: we are very uncertain about the estimate.

fGRADE: The Grading of Recommendations Assessment, Development and Evaluation working group.

Acu, acupuncture; CHM, Chinese Herbal Medicine; SMD, standard mean difference; WM, western medicine. 
Table 2 Subgroup analysis on the duration of treatment

\begin{tabular}{|c|c|c|c|}
\hline Subgroup analysis & $\begin{array}{l}\text { Number of studies (number } \\
\text { of patients) }\end{array}$ & SMD, $95 \% \mathrm{Cl}$ & P interaction \\
\hline \multicolumn{4}{|l|}{ Improvement in dependency } \\
\hline Short duration ( $\leq 1$ month) & $17(1980)$ & $0.92(0.58$ to 1.26$)$ & 0.43 \\
\hline Longer term duration (>1 month) & $10(781)$ & $1.12(0.75$ to 1.5$)$ & \\
\hline \multicolumn{4}{|l|}{ Improvement in motor function } \\
\hline Short duration ( $\leq 1$ month) & $20(2541)$ & 0.71 (0.40 to 1.02$)$ & 0.66 \\
\hline Longer term duration (>1 month) & $6(558)$ & 0.85 (0.33 to 1.36$)$ & \\
\hline \multicolumn{4}{|l|}{ Improvement in depression } \\
\hline Short duration ( $\leq 1$ month) & $9(809)$ & $-1.19(-1.62$ to to 0.76$)$ & 0.23 \\
\hline Longer term duration (>1 month) & $6(466)$ & $-0.76(-1.31$ to 0.21$)$ & \\
\hline \multicolumn{4}{|l|}{ Improvement in swallowing function } \\
\hline Short duration ( $\leq 1$ month) & $10(801)$ & $-0.66(-1.02$ to 0.30$)$ & 0.60 \\
\hline Longer term duration (>1 month) & $3(273)$ & $-0.53(-0.86$ to 0.20$)$ & \\
\hline
\end{tabular}

SMD, standard mean difference.

\section{Improvement in swallowing function}

Six RCTs and two quasi-RCTs demonstrated the superiority of the effect of acupuncture and WM combined therapy compared with baseline WM therapy (639 patients; SMD $-0.78,95 \%$ CI -1.22 to $-0.34 ; \mathrm{I}^{2}=86 \%$, low quality, figure 5 and table 1). There were no studies in the subgroups that compared the effect of CHM and WM combined therapy with baseline WM therapy in the improvement of swallowing function.

\section{Subgroup analysis}

A subgroup analysis was conducted based on short-term treatment duration $(\leq 1$ month) versus longer term treatment duration ( $>1$ month) for all outcomes. The results indicate no subgroup effects in all outcomes. The details are shown in table 2 and online supplemental figures 9-12.

\section{DISCUSSION}

Overall, the systematic review provided evidence that the combined use of CHM or acupuncture with WM might have more benefits than WM therapy alone in stroke rehabilitation. However, these results should be interpreted cautiously given some concerns associated with potential publication bias and low quality of several original studies, randomisation in allocation and the short duration of the treatment periods. Subgroup analysis suggested that acupuncture combined with WM may be more efficacious together than alone in different outcomes. Our results also indicate that acupuncture combined with WM has a stronger effect than CHM combined with WM and has the potential for more extensive use in stroke rehabilitation.

Despite there are existing systematic reviews on the effect of CHM and acupuncture in managing stroke, our review offers various new perspectives. First, regarding our methodology, we only included papers of RCTs and quasi-RCTs with a sample size of 50 or more. Stricter inclusion criteria could increase the quality of evidence and reduce the risk of bias. Second, we focused on evaluating outcomes related to stroke rehabilitation that have not been thoroughly discussed previously. For instance, efficacy in improving swallowing and poststroke depression have not been analysed in existing reviews. ${ }^{420}$ These complications are known to affect the quality of life of poststroke patients, particularly swallowing function, which increases the rate of institutionalisation and mortality. ${ }^{21}{ }^{22}$ Third, we reported on subgroup analysis based on the treatment characteristics of CHM versus acupuncture. Such joint comparisons between CHM and acupuncture have not previously been attempted. Additionally, we indicated the most commonly used acupuncture points and herbs in our analysis and listed them in our baseline characteristic table (online supplemental table 2).

Our results hint that CHM and acupuncture combined with WM may be more efficacious than WM alone in terms of some aspects, which may need further investigation using RCTs. Combined WM therapy with CHM appears to be better for improving depression than combined WM therapy with acupuncture. Combined WM treatment with acupuncture instead has a greater effect on improving dependency and motor function than that with CHM. However, it is known that patients with depression are sensitive to psychological stimuli and their motor function and dependency are affected by positive motivation. As acupuncture positively impacts both of these aspects, we may also need to consider all these effects. Thus, the results from our subgroup analysis require further confirmation through direct comparisons between CHM and acupuncture, either using statistical models such as network meta-analysis or future clinical trials. Such evidence would be very valuable to medical providers in 
choosing the best therapy to improve different outcomes of patients with stroke.

This systematic review has several limitations. The first major limitation is the quality of the included studies. Regarding selection bias, 36 included studies had low risk of bias, 15 had unclear risk of bias and 7 had high risk of bias. This may lead to an inaccurate conclusion on the effects of CHM and acupuncture. Second, some of the effects observed in our study may be a result of publication bias, and most included studies exhibited reporting bias. The resulting asymmetrical funnel plots in the improvement of motor function and improvement of swallowing function measured by water drinking test suggest an overestimation of effect sizes in some of the included trials due to selective reporting. Third, heterogeneity among the studies was relatively high, which may affect the quality of results generated by meta-analysis. Fourth, there was a lack of a placebo control in the included studies as the intervention designs were predominantly CHM or acupuncture plus WM in the treatment groups versus WM only in the control groups. These designs could not negate any placebo effects produced by CHM or acupuncture, which may have potentially induced an overestimation of the treatment effect in these studies. Finally, we did not conduct subgroup analyses on different ages, genders and severities in our outcome assessment.

\section{CONCLUSIONS}

The combined use of CM and WM may have positive effects in stroke rehabilitation compared with the use of WM therapy alone. This study will guide the enhancement of rigour and reduction of bias in future clinical trials to better validate the effects of $\mathrm{CM}$ and acupuncture. Further studies with longer durations of treatment and follow-ups should be conducted with more rigorous designs.

\section{Author affiliations}

${ }^{1}$ Hong Kong Chinese Medicine Clinical Study Centre, School of Chinese Medicine, Hong Kong Baptist University, Hong Kong SAR, China

${ }^{2}$ Department of Medicine and Therapeutics and Hong Kong Institute of Integrative Medicine, Prince of Wales Hospital, Faculty of Medicine, The Chinese University of Hong Kong, Hong Kong SAR, China

${ }^{3}$ Department of Health Research Methods, Evidence and Impact, McMaster University, Hamilton, Ontario, Canada

${ }^{4}$ Clinical Laboratory, Shanghai Traditional Chinese Medicine Integrated Institute of Vascular Abnomalies, Shanghai, China

${ }^{5}$ Institute of Medical Research, Northwestern Polytechnical University, Xi'an, Shanxi, China

${ }^{6}$ Evidence Based Medicine Center, School of Basic Medical Sciences, Lanzhou University, Lanzhou, China

${ }^{7}$ School of Chinese medicine, Hong Kong Baptist University, Hong Kong SAR, China

${ }^{8}$ Brain Center, Guangdong Procincial Hospital of Traditional Chinese Medicine,

Guangdong University of Chinese Medicine, Guangzhou, China

${ }^{9}$ The Nethersole School of Nuring, Faculty of Medicine, the Chinese University of Hong Kong, Hong Kong SAR, China

${ }^{10}$ Department of Medicine, Li Ka Shing Faculty of Medicine, the University of Hong Kong, Hong Kong SAR, China

${ }^{11}$ Department of Rehabilitation Sciences, Hong Kong Polytechnic University, Hong Kong SAR, China
${ }^{12}$ Hong Kong Association for Integration of Chinese-Western Medicine Limited, Hong Kong SAR, China

Contributors LLDZ and YZ screened all articles and drafted the manuscript. They contributed equally and could be considered as co-first authors. NW, XW, TS conducted the literature searching and synthesised the data. ZL, HL extracted the data and manufactured the figures. NW and LY worked independently and assessed potential risks of bias for all included studies using the Cochrane's tool for assessing risk of bias and they also help to make the tables. CSY and JG contributed to revise the manuscript. AL, ECY designed the research plan and gave supervision. SL, JC, KWC, BFLN, ZB contributed to monitor the research and give critical comments and finalised the manuscript.

Funding This study was funded by Innovative Technology Commission of the government of Hong Kong SAR (GSP/008/18).

Competing interests None declared.

Patient consent for publication Not required.

Provenance and peer review Not commissioned; externally peer reviewed.

Open access This is an open access article distributed in accordance with the Creative Commons Attribution Non Commercial (CC BY-NC 4.0) license, which permits others to distribute, remix, adapt, build upon this work non-commercially, and license their derivative works on different terms, provided the original work is properly cited, appropriate credit is given, any changes made indicated, and the use is non-commercial. See: http://creativecommons.org/licenses/by-nc/4.0/.

ORCID iDs

Linda LD Zhong http://orcid.org/0000-0002-3877-1914

Janita Chau http://orcid.org/0000-0002-3750-7396

\section{REFERENCES}

1 Fang J, Chen L, Chen L, et al. Integrative medicine for subacute stroke rehabilitation: a study protocol for a multicentre, randomised, controlled trial. BMJ Open 2014;4:e007080.

2 Wang J, Xiong X. Current situation and perspectives of clinical study in integrative medicine in China. Evid Based Complement Alternat Med 2012;2012:1-11.

3 Tang J-L, Liu B-Y, Ma K-W. Traditional Chinese medicine. Lancet 2008;372:1938-40.

4 Junhua Z, Menniti-Ippolito F, Xiumei G, et al. Complex traditional Chinese medicine for poststroke motor dysfunction: a systematic review. Stroke 2009;40:2797-804.

5 Gaire BP. Herbal medicine in ischemic stroke: challenges and prospective. Chin J Integr Med 2018;24:243-6.

6 Yang A, HM W, Tang JL. Acupuncture for stroke rehabilitation. Cochrane Database Syst Rev 2016;2016:CD004131.

7 Hung CY-F, Wu X-Y, Chung VC-H, et al. Overview of systematic reviews with meta-analyses on acupuncture in post-stroke cognitive impairment and depression management. Integr Med Res 2019;8:145-59.

8 Xu M, Li D, Zhang S. Acupuncture for acute stroke. Cochrane Database Syst Rev 2018;3:CD003317.

9 Moher D, Liberati A, Tetzlaff J, et al. Preferred reporting items for systematic reviews and meta-analyses: the PRISMA statement. BMJ 2009;339:b2535.

10 Wang H-L, Zeng H, Xu M-B, et al. Efficacy and safety of Chinese herbal medicine for primary intracerebral hemorrhage: a systematic review of randomized controlled trials. Front Pharmacol 2019;10:1139.

11 Cai W, Mueller C, Li Y-J, et al. Post stroke depression and risk of stroke recurrence and mortality: a systematic review and metaanalysis. Ageing Res Rev 2019;50:102-9.

12 Huang J, Shi Y, Qin X, et al. Clinical effects and safety of electroacupuncture for the treatment of poststroke dysphagia: a comprehensive systematic review and meta-analysis. Evid Based Complement Alternat Med 2020;2020:1-9.

13 Lee MS, Shin B-C, Kim J-ILee JA, Kim TH, et al. Moxibustion for stroke rehabilitation: systematic review. Stroke 2010;41:817-20.

14 Zhang HW, Zheng R, Xu C. Tai chi for improving recovery after stroke. Cochrane Database Syst Rev 2018;3:CD010207.

15 Abdul Rani AA, Ab Ghani RM, Shamsuddin S. Massage therapy for improving functional activity after stroke. Cochrane Database Syst Rev 2015;10:CD011924.

16 Ouzzani M, Hammady H, Fedorowicz Z, et al. Rayyan-a web and mobile APP for systematic reviews. Syst Rev 2016;5:210. 
17 Shuster JJ. Review: cochrane Handbook for systematic reviews for interventions, version 5.1.0, published 3/2011. Julian P.T. Higgins and Sally green, editors. Res Synth Methods 2011;2:126-30.

18 Xin Z, Xue-Ting L, De-Ying K. Grade in systematic reviews of acupuncture for stroke rehabilitation: recommendations based on high-quality evidence. Sci Rep 2015;5:16582.

19 Guyatt GH, Oxman AD, Vist GE, et al. Grade: an emerging consensus on rating quality of evidence and strength of recommendations. $B M J$ 2008;336:924-6.
20 Wu B, Liu M, Liu H, et al. Meta-Analysis of traditional Chinese patent medicine for ischemic stroke. Stroke 2007;38:1973-9.

21 Arnold M, Liesirova K, Broeg-Morvay A, et al. Dysphagia in acute stroke: incidence, burden and impact on clinical outcome. PLoS One 2016;11:e0148424.

22 Beharry A, Michel P, Faouzi M, et al. Predictive factors of swallowing disorders and bronchopneumonia in acute ischemic stroke. J Stroke Cerebrovasc Dis 2019;28:2148-54 